\title{
Two Distinct Types of Natural Selection in Turbidostat-like and Chemostat-like Ecosystems
}

\author{
JAROSLAV FLEGR* \\ Department of Parasitology and Hydrobiology, Faculty of Science, Charles University, \\ Vinicna 7, CZ-128 44 Prague, Czech Republic
}

(Received on 26 March 1997, Accepted on 17 April 1997)

\begin{abstract}
The size stability of natural populations indicates that natality and mortality rates are on average equal. Fine tuning of the two independent processes suggests the existence of a regulating mechanism. Two types of such mechanisms exist. A decrease in the number of organisms can either speed up the natality rate or slow down the mortality rate. The former mechanism (chemostat-like) acts whenever the population growth is limited by the concentration of a resource. The latter (turbidostat-like) could be suspected whenever the population growth is limited by predators or parasites. Simulation experiments showed that under chemostat-like and turbidostat-like conditions, organisms are selected toward efficiency (grams of biomass produced/grams of resource consumed) and natality rate (grams of biomass/time), respectively. The existence of two types of selection and the nature of parameters that are selected to recall the old idea of $\mathrm{r}-\mathrm{K}$ strategies. The main difference is that while the old model predicts the existence of $\mathrm{r}-\mathrm{K}$ continuum, this model shows that the two strategies are exclusive.
\end{abstract}

(C) 1997 Academic Press Limited

\section{Introduction}

The idea of $\mathrm{r}$ and $\mathrm{K}$ strategies ( $\mathrm{r}$ and $\mathrm{K}$ selection) was very popular among students of ecology. Originally $r$-selection meant selection for high population growth in uncrowded populations and $\mathrm{K}$-selection referred to selection for competitive ability in crowded populations (MacArthur \& Wilson, 1967; Hairston et al., 1970; Wilbur et al., 1974). Later, however, the meaning of these terms was broadened (Pianka, 1970, 1972; Southwood, 1977) and partially changed (Stearns, 1976, 1977; Parry, 1981).

Currently, r-strategists are considered those organisms that invest their reproductive effort into the production of a great number of offspring. Biological qualities of the offspring, reflected by the chance that an individual will survive and reproduce, can be low. On the contrary, the K-strategists invest their reproductive effort into the production of fewer

*E-mail: FLEGR@MBOX.CESNET.CZ offspring with higher parental investment per capita. Consequently, the chance that an individual will survive and reproduce can be relatively high. The biological properties of $\mathrm{r}$-strategists and $\mathrm{K}$-strategists can also be characterized in concrete terms like body size, generation time, number of reproductive seasons during the lifespan (all lower in r-strategists), or by the effect of the strategy on the properties of the whole population. Populations of r-strategists fluctuate with time. Their size is usually lower than the maximal carrying capacity of the environment. Competition in such populations is low or non-existent. The individuals are often eliminated from the population randomly, and there is only a low correlation between the biological qualities or the age of individuals and their actual probability of dying. The properties of populations of K-strategists are just the opposite. r-strategy is often considered an adaptation for living in unstable or unpredictable environments, e.g., resource-rich ecosystems in the earliest stages of ecological succession. On the other hand, K-strategy is considered the optimal adaptation 
for living in stable or predictable environments, for old and crowded ecosystems in or near a climax stage.

The idea of $\mathrm{r}-\mathrm{K}$ strategies was introduced by MacArthur. The names $\mathrm{K}$ and $\mathrm{r}$ have been derived from names of two parameters in the common form of the logistic equation

$$
\mathrm{d} N / \mathrm{d} t=r N(1-N / K)
$$

where $r$ is the intrinsic growth rate of the population (under optimal conditions) and $K$ is the carrying capacity of the environment (the size of population for which

$$
\mathrm{d} N / \mathrm{d} t=0)
$$

The theory in its original formulation (MacArthur, 1962), as well as its later development (MacArthur \& Wilson, 1967), suggests that the existence of two different strategies has something to do with the logistic equation-based model of population growth. As it has already been pointed out (Pianka, 1972; Ginzburg, 1992), this notion is false. In the systems described by the logistic equation, both parameters $r$ and $K$ are simultaneously under pressure from natural selection. From the equation, it cannot be explained why two distinct ecological strategies, rather than a single mixed one, should exist. Moreover, the logistic equation represents a phenomenological (descriptive) rather than mechanistic model. It is a rearranged second-order polynomial $\mathrm{d} N / \mathrm{d} t=a N-b N^{2}$. This function fits a S-shaped growth curve usually obtained in experiments, but it does not deal with the actual mechanism of growth and death in natural populations.

It can be concluded that despite the popularity of the $\mathrm{r}-\mathrm{K}$ idea and despite the evident existence of two distinct ecological strategies, no theoretical model exists so far which could explain the r-K dichotomy phenomenon.

The present study attempts to show that the existence of two basic types of selection, and consequently two basic types of ecological strategies, can be explained by the existence of just two number-regulating mechanisms that can control the growth of a population. Because any population is subjected to one of these two mechanisms, it is also forced to adopt one of two exclusive life strategies.

\section{Results}

\subsection{MODEL OF TWO TYPES OF NUMBER-REGULATING MECHANISMS}

Despite the existence of temporal fluctuations, the long-term size of a population of different biological species is mostly stable. Such stability implies that natality and mortality rates in natural populations are essentially equal. Such fine tuning of two relatively independent processes in an unpredictable and fluctuating environment is not possible without a negative feedback loop-based number-regulating mechanism.

Basically, there are only two possibilities for the realization of such a mechanism. An increase (decrease) of population number must either induce a decrease (increase) in the natality rate or an increase (decrease) in the mortality rate. Regardless of the type of number-regulating mechanism, a size of the population exists, for which the natality and mortality rates are equal. Under normal conditions this equilibrium point is stable, i.e., after a disturbance the size of the population returns to its original value.

From ecological and evolutionary points of view there is an important difference between these two number-regulating mechanisms. It can be shown that the type of regulation mechanism determines which property of living systems actually contributes to their fitness, i.e., which is subjected to natural selection.

The mortality rate-regulation can be modeled in a laboratory turbidostat (a flow reactor). In this system for continuous cultivation of microorganisms, the population size is regulated by a negative feedback loop between population size (usually monitored by optical density of the cultivation medium in a tank) and a rate of the pumping of medium through (and consequently a rate of the washing-out of the microorganisms from) the tank. The growth of microbes can be described by a differential equation:

$$
\mathrm{d} N / \mathrm{d} t=N(r-D N)
$$

where $N, t$ and $r$ are population size, time and individual growth rate (natality minus natural mortality per capita), respectively. $D$ is a constant, a technical parameter of the turbidostat, which determines the rate of the pumping of the cultivation medium through the cultivation tank when population size is equal to one.

The second type of number-regulating mechanism operates in a chemostat. This system for continuous cultivation of microorganisms can be realized in any turbidostat by keeping the concentration of some growth factor in the inflow medium at very low limits. Under these conditions the growth of microorganisms and the dynamics of the concentration of the limiting component in the medium (limiting resource) can be described by:

$$
\begin{gathered}
\mathrm{d} N / \mathrm{d} t=N(\operatorname{Min}(r, R I k)-D N) \\
\mathrm{d} R / \mathrm{d} t=D N S-D N R-N \operatorname{Min}(r / k, R I)
\end{gathered}
$$


where $R$ and $S$ are concentrations of the resource in the cultivation tank and in the inflow medium, respectively, $k$ is the efficiency [the number of individuals produced per units of resource consumed (in the chemostat literature this quantity is called "yield")], and $I$ is the rate of input of resource into one organism (per capita consumption rate) under conditions when $R=1 . r$ is an individual growth rate under the conditions of optimal concentration of the resource. The meanings of all other symbols are the same as in the previous model. This proposed model differs from common models of the chemostat (Tilman, 1982) in two respects. First, the proposed model is universal; it can operate in the chemostat, as well as in the turbidostat mode. Second, the growth of organisms is described by a non-smooth function $\operatorname{Min}(r, R I k)$, rather then by a Monod's function $r$ $R /(R+K)$ [in Monod's function (Monod, 1950) the $K$ is a half-saturation constant, the concentration of resource at which growth rate reaches half its maximal value]. The Minimum function (the simplest function for numerical modeling) is preferred not only from pragmatic reasons. It also better reflects the behavior of real organisms. When the concentration of a resource increases, the Monod function only asymptotically approaches a theoretical maximal growth rate. For real organisms, however, the maximum growth rate exists, which can be reached under the condition of optimal resource concentration but cannot be exceeded, no matter any further increase in the resource concentration (Peczurkin, 1981). In any case, a type of function has no influence on a general behavior of the studied systems.

In the proposed model four types of variables exist: (1) output variables $(N, R)$; (2) technical parameters of the device $(S, D)$; (3) elementary biological parameters of organisms $(r, k)$; and (4) composite parameter of the organisms $(I)$. In contrast to the elementary parameters $k$ and $r$, the composite parameter $I$ cannot be a criterion of fitness. It represents either a physical constant reflecting the coefficient of diffusion of resource particles (Flegr, 1990), or the result of a physiological regulation that optimizes the rate and efficiency of growth for a current resource concentration (Shnol, 1979).

\subsection{COMPETITION IN TURBIDOSTATIC AND CHEMOSTATIC SYSTEMS}

To study the competition in the number-regulated populations one must simulate the dynamics of a system of two different species (A and B). Such systems can be described by:

$$
\begin{aligned}
& \mathrm{d} N_{\mathrm{A}} / \mathrm{d} t= N_{\mathrm{A}}\left(\operatorname{Min}\left(r_{\mathrm{A}}, R I k_{\mathrm{A}}\right)-D\left(N_{\mathrm{A}}+N_{\mathrm{B}}\right)\right) \\
& \mathrm{d} N_{\mathrm{A}} / \mathrm{d} t= N_{\mathrm{B}}\left(\operatorname{Min}\left(r_{\mathrm{B}}, R I k_{\mathrm{B}}\right)-D\left(N_{\mathrm{A}}+N_{\mathrm{B}}\right)\right) \\
& \mathrm{d} R / \mathrm{d} t=D\left(N_{\mathrm{A}}+N_{\mathrm{B}}\right) S-D\left(N_{\mathrm{A}}+N_{\mathrm{B}}\right) R-N_{\mathrm{A}} \\
& \operatorname{Min}\left(r_{\mathrm{A}} / k_{\mathrm{A}}, R I\right)-N_{\mathrm{B}} \operatorname{Min}\left(r_{\mathrm{B}} / k_{\mathrm{B}}, R I\right)
\end{aligned}
$$

One can analyse the competition of two species that differ in individual growth rates as well as in efficiencies. It is illustrative to suppose that species A has a higher individual growth rate and lower efficiency than species $B$. The result of numeric simulation of competition under conditions of different resource concentrations in the inflow medium is shown in Fig. 1. Evidently three different situations could occur.

(a)

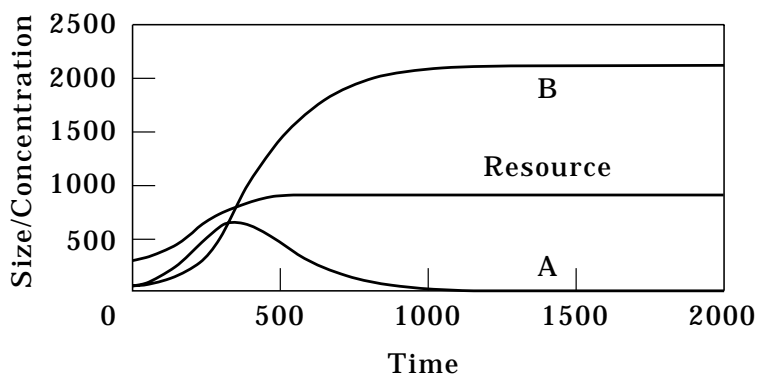

(b)

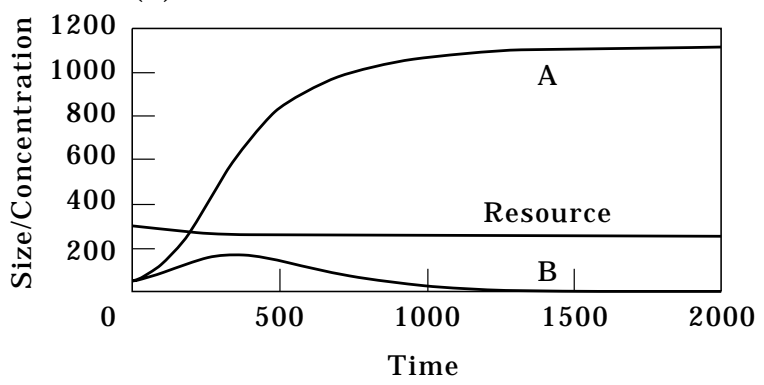

(c)

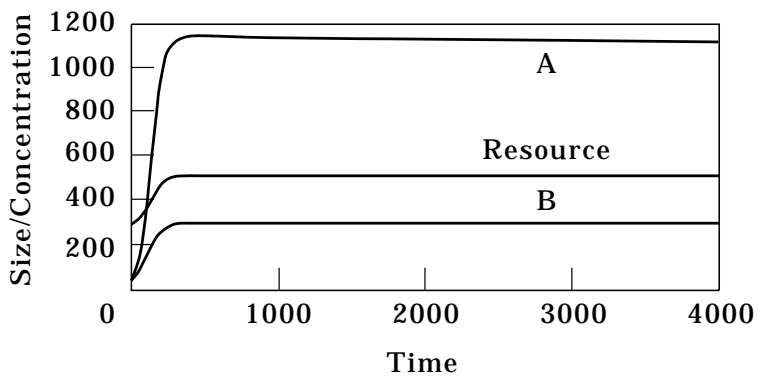

FIG. 1. Simulation of the competition of two species, A and B under conditions of different concentrations of resource $S$ in the inflow medium. The simulation was done using Delphi 2.0 compiled program POPULACE (available at http://natur.cuni.cz/ $\sim$ flegr/PROGRAMS) with parameters: $D=0.0007, I=0.00001$, $r_{\mathrm{A}}=0.1, r_{\mathrm{B}}=0.15, k_{\mathrm{A}}=30, k_{\mathrm{B}}=20, N_{\mathrm{A}}(0)=50, N_{\mathrm{B}}(0)=50$ and $R(0)=300$. Concentration of the resource in the inflow medium $(S)$ was 1000, 300 and 553 arbitrary units for Fig. 1(a), (b) and (c), respectively. 
(1) When the concentration of the resource is low, the growth of both populations is regulated by the chemostatic mechanism. The species with lower efficiency (species A) is completely displaced after an initial period of growth.

(2) When the concentration of the resource is sufficiently high, the opposite situation occurs. Both populations are regulated by the turbidostatic mechanism, which results in the displacement of the population with a lower individual growth rate (species B) after the initial period of growth.

(3) A range of concentrations of the resource also exists for which the population of species A is regulated by the chemostatic mechanisms while the population of species $\mathrm{B}$ is regulated by the turbidostatic mechanism. A long-term coexistence of two populations is then possible. During the period of coexistence, species $\mathrm{A}$ is being selected toward a higher efficiency of growth while species B toward a higher maximal growth rate. This selection could result either in the switching of the chemostatic regulation of population A to the turbidostatic, or in the switching of the turbidostatic regulation of population B to the chemostatic. After such an event, the second species is quickly displaced. However, this event might be quite rare, therefore, the equilibrium is rather stable and the conditions for coexistence are broad. For example, Fig. 2 shows that the range of concentrations of resource in which both species can coexist is relatively broad.

It is very interesting and even contraintuitive that the resource equilibrium concentration does not depend on concentration of the resource in the inflow medium and on relative sizes of both populations in the cultivation tank. This equilibrium concentration $R_{\mathrm{E}}$ can be computed

$$
R_{\mathrm{E}}=r_{\mathrm{A}} /\left(k_{\mathrm{B}} I\right) .
$$

In summary the chance of an organism as survival in interspecies competition is determined by two basic biological parameters (individual growth rate and the efficiency) and by the type of number-regulating mechanism in operation. In a long-term perspective it is also determined by the organism's capacity to improve these parameters in response to natural selection (by evolutionary constrains).

\section{Discussion}

Results of our simulation experiments showed that under chemostat-like cultivation conditions, the efficiency was the critical factor in the fitness of an organism and also a subject of natural selection.
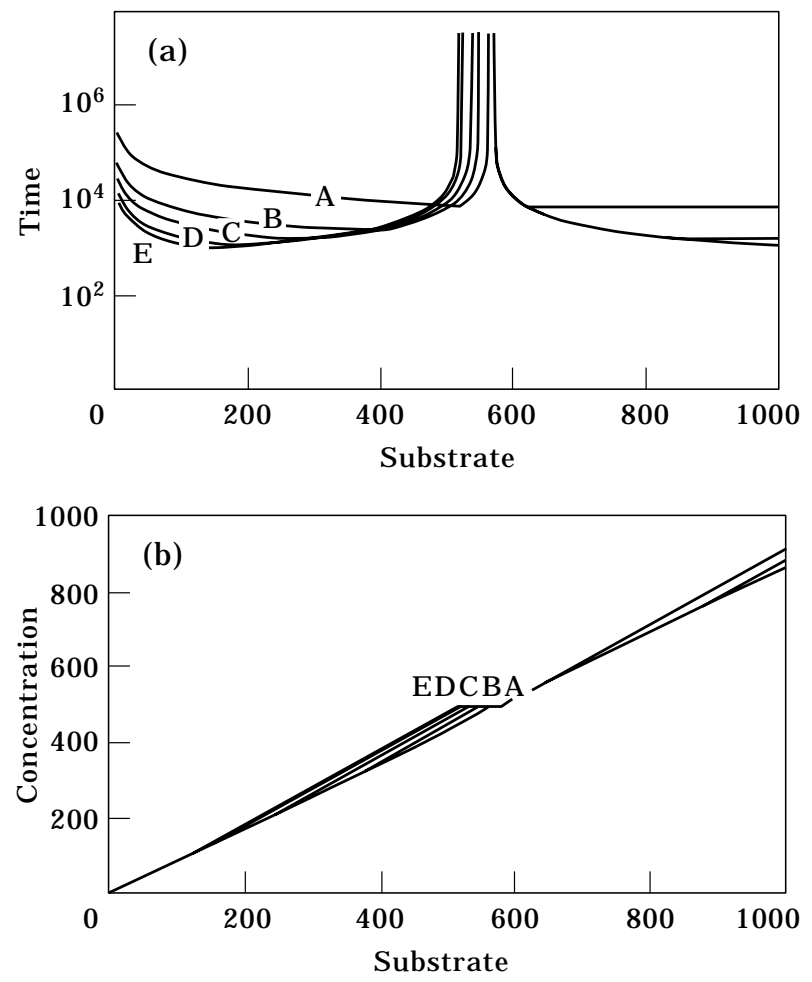

FIG. 2. Influence of a concentration of resource $S$ in the inflow medium on the time of competitive exclusion of less fitted species (a) and on the concentration of the resource in the cultivation tank in the time of the exclusion (b). Five different competition experiments $\mathrm{A}, \mathrm{B}, \mathrm{C}, \mathrm{D}$ and $\mathrm{E}$ were simulated $\left(\mathrm{A}: r_{\mathrm{B}}=0.11\right.$, $k_{\mathrm{A}}=22 ; \mathrm{B}: r_{\mathrm{B}}=0.15, k_{\mathrm{A}}=30 ; \mathrm{C}: r_{\mathrm{B}}=0.2, k_{\mathrm{A}}=40 ; \mathrm{D}: r_{\mathrm{B}}=0.3$, $\left.k_{\mathrm{A}}=60 ; \mathrm{E}: r_{\mathrm{B}}=0.4, k_{\mathrm{A}}=60\right)$. Values of all other parameters in all experiments were as in Fig. 1. In any experiment 200 simulation runs were done for concentrations $(S)$ between 5 and 1000 arbitrary units. The simulation run was stopped when the number of organisms of one species decreased under one or after $10^{7}$ time units of the species coexistence.

Under the turbidostat-like conditions the critical factor was the individual growth rate.

It seems that in biological systems, even in the laboratory ones, a broad spectrum of properties of the organisms plays an important role in interspecies and intraspecies competition. For example, mutants that can stick to the walls of the cultivation tank or that can replace part of the resource with another component of the medium will displace their competitors. Such mutations could be considered an improvement of growth rate and an improvement of efficiency in the turbidostat and the chemostat, respectively. Consequently, they will be selected for under both turbidostat-like and chemostat-like conditions. When the same mutation can be interpreted as the improvement of growth rate or efficiency according to the type of number-regulating mechanism in operation, the practical meanings of discrimination between the two basic parameters of growth (rate and efficiency) might be questioned. 
The reason for such discrimination is that a category of mutations exists for which the improvement of the growth rate can be achieved only at the expense of the efficiency (and vice versa). The destiny of these mutations is determined by the type of number-regulating mechanism in operation. The trade off between efficiency and rate could be a very common phenomenon in biological systems (Shnoll, 1979). It means that these mutations can be numerous and their destiny could determine the life strategy of organisms and the character of evolution of the population or of the species.

The present model describes an artificial system for the continual cultivation of microorganisms. The chemostat-like as well as turbidostat-like types of number-regulating mechanisms also operate in natural ecosystems. The chemostat-like mechanisms operate whenever the growth of population is being limited by an availability of some resource (energy, substance, space). The turbidostat-like mechanisms must be suspected whenever the growth of a population is being limited by the activities of predators or parasites.

Real populations are continuously under influence from many factors. Their growth is controlled by the availability of different resources and by the activities of different predators. Their growth should be described by a general expression:

$$
\mathrm{d} N / \mathrm{d} t=\mathrm{f}\left(N^{\mathrm{A}}, N^{\mathrm{B}}, N^{\mathrm{C}}, \ldots N^{\mathrm{Z}}\right)
$$

Here, more than one value of $N$ can exist for which the $\mathrm{d} N / \mathrm{d} t=0$. Only some of these equilibrium points, of course, can be stable. The population can move from one equilibrium point to another. At any moment, however, the population can be at only one equilibrium point. For any equilibrium point there are always principal components that are responsible for the return of the population size to the original value after a fluctuation, i.e., for the negative feedback loop. The character of these principal components determines what type of number-regulating mechanism is functioning at any particular equilibrium point.

When we look at the list of properties of $\mathrm{r}$-strategists and K-strategists given in the Introduction or elsewhere (Pianka, 1978; Parry, 1981), we can see that the properties typical for r-strategists are always more or less tightly connected with maximization of the individual growth rate, while for $\mathrm{K}$-strategists with the maximization of the efficiency. Evidently, r-strategists are typical inhabitants of resource-rich ecosystems while $\mathrm{K}$-strategists of crowded ecosystems where the limitation by the unavailability of some resource can be observed or expected. This suggests that the r-strategists and the $\mathrm{K}$-strategists could in fact be the organisms living under the turbidostat-like and chemostat-like conditions, respectively. The $\mathrm{r}$ strategists are being selected toward a higher $r$ (toward maximal growth rate) which forces them to adopt the $r$-maximizing strategies. At the same time, the K-strategists are being selected toward a maximal efficiency of growth (maximal k) which forces them to adopt the $k$-maximizing strategy.

Three differences between $\mathrm{r}-\mathrm{K}$ and $r-k$ conceptions should be pointed out:

(1) The $r-k$ conception predicts that the maximal growth rate and the efficiency are being maximized during evolution in turbidostat and chemostat, respectively.

According to $\mathrm{r}-\mathrm{K}$ theory, parameters maximized are growth rate and carrying capacity of environment at the turbidostat and chemostat, respectively. From the results of simulation experiments it is evident, however, that the phenomenologically defined constant $K$ (see the definition) is being maximized both in the turbidostat and the chemostat.

(2) Ecologists usually suppose that instead of distinct $\mathrm{r}$ or $\mathrm{K}$ selection, the $\mathrm{r}-\mathrm{K}$ selection continuum normally exists (Pianka, 1978). Our results show that these two types of selection are not only distinct, but also exclusive. The $\mathrm{r}-\mathrm{K}$ continuum, which can be observed in nature, can rather be interpreted as the result of an existence of evolutionary constraints.

(3) The $\mathrm{r}-\mathrm{K}$ theory is based on empirical data only. There is no theoretical model that could explain the existence of these two types of selection. On the other hand, $r-k$ conception, which explains the same empirical data, was deduced from a mechanistic model of population growth.

I would like to thank Vladimir Korinek and Anton Markos for cogent criticisms to the manuscript. This work has been supported by grant 206/95/0638 from the Grant Agency of the Czech Republic.

\section{REFERENCES}

FLEGR, J. (1990). Does a cell perform isoelectric focusing? BioSystems 24, 127-133.

GinZBURG, L. R. (1992). Evolutionary consequences of basic growth equations. Trends Ecol. Evol. 7, 133.

Hairston, N. G., Tinkle, D. W. \& Wilbur, H. M. (1970). Natural selection and the parameters of population growth. J. Wildl. Manage. 34, 681-690.

MacArthur, R. H. (1962). Some generalized theorems of natural selection. Proc. Nat. Acad. Sci. U.S.A. 48, 1893-1897.

MacArthur, R. H. \& Wilson, E. O. (1967). The Theory of Island Biogeography. Princeton, NJ: Princeton University Press. 
Monod, J. (1950). La technique de culture continue; theorie et applications. Annals de l'Institut Pasteur 79, 390-410.

PARry, G. D. (1981). The meanings of r- and K-selection. Oecologia (Berlin) 48, 260-264.

Peczurkin, N. S. ed. (1981). Smeshanyje Protocznyje Kultury Mikroorganizmov. (Mixed Continuous Cultures of Microorganisms). Novosibirsk: Nauka Press.

Pianka, E. R. (1970). On r- and K-selection. Amer. Natur. 104, 592-597.

Pianka, E. R. (1972). r and K selection or b and d selection? Amer. Natur. 106, 581-588.

Pianka, E. R. (1978). Evolutionary Ecology. 2nd Edn. New York: Harper \& Row Publishers.

Shnoll, S. E. (1979). Fiziko-chimiczeskije Faktory Biologiczeskoj
Evoljucii. (Physico-chemical Factors of Biological Evolution). Moskva: Nauka Press.

Southwood, T. R. E. (1977). Habitat, the templet for ecological strategies? J. Anim. Ecol. 46, 337-365.

Stearns, S. C. (1976). Life history tactics, a review of the ideas. O. Rev. Biol. 51, 3-47.

STEARns, S. C. (1977). The evolution of life history traits, a critique of the theory and a review of the data. Annu. Rev. Ecol. Syst. 8, 145-171.

Tilman, D. (1982). Resource Competition and Community Structure. Princeton: Princeton University Press.

Wilbur, H. M., Tinkle, D. W. \& Collins, J. P. (1974). Environmental certainty, trophic level, and resource availability in life history evolution. Amer. Natur. 108, 805-817. 